\title{
A CASE OF "MIOTONIA CONGENITA."
}

By W. H. HAYNES, M.D.,

Attending Neurologist to the Central Throat Hospital and Polyclinic: Assistant Physician, Neurological Department, Brooklyn Eye and Ear Hospital: Member of the Brooklyn Netrological society, etc., Brooklyn. N. Y.

The following case is described under the above heading, more on account of the clinical phenomena than for the accuracy of the nomenclature. The patient was presented to the Brooklyn Neurological Society at its meeting in February, 1897, and the opinion expressed by all was that they had never seen a similar case, and were unable to classify it.

Peter C., aged 28, born in Pennsylvania, single, and by occupation a laborer. His father died, aged 98 ; mother still alive, aged 72. She had six children, two of whom died in infancy. Some of her near relatives have died of phthisis.

$\mathrm{He}$ is strongly addicted to alcohol and tobacco, denies, and there is no evidence of, any venereal diseases. When a boy he was in the habit of staying in the vater of a pond on his father's farm all day diving and remaining under water for a long time, thereby in one instance nearly losing his life by drowning, and to this habit his people ascribe the source of his present disorder. It was first noticed when he began attending school at the age of seven years. His teachers then observed the difficulty he had in pronouncing words. At fourteen he could protrude his tongue but half-way, so that lis parents thought he was tongue-tied and tried to have the phrenum cut, but were informed it would do no good, as it was not the cause of the difficulty. He has never had any illness, with the exception of being laid up for five weeks greatly prostrated after the drowning accident before mentioned, and excepting, at I6 years of age, fracture of both bones of right forearm.

Six or seven years ago, following a debauch, he had an attack of remittent fever and bronchitis, accompanied 
by hoarseness, which has continued with increased difficulty of speech, better or worse, according as he had or had not a cold.

Two years later, following an attack of so-called intermittent typhoid fever and grippe, which lasted seven weeks, and which was also preceded by a heavy debauch,

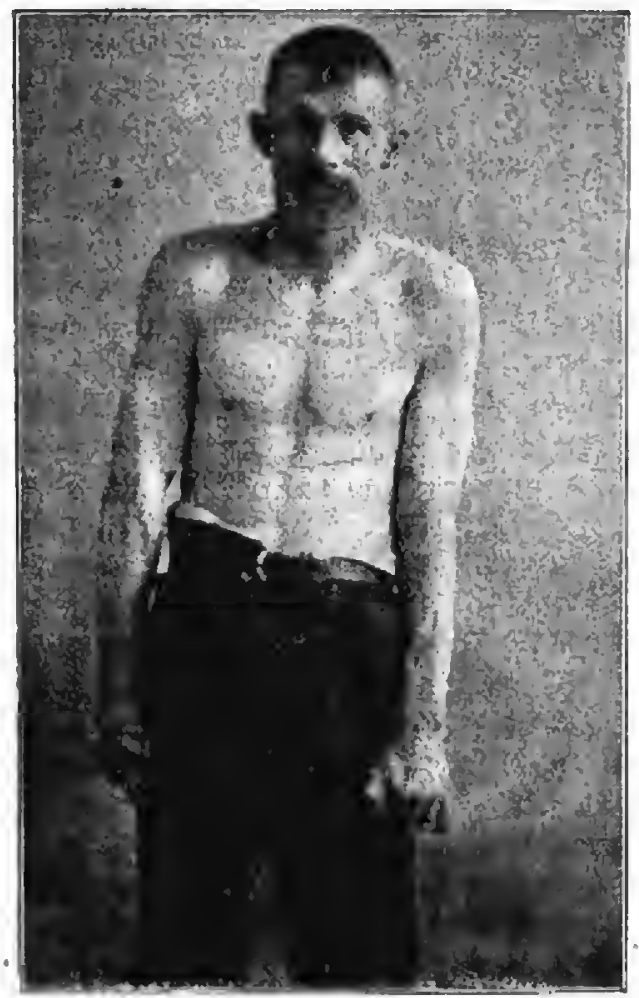

FIG. No. I.

it was noticed that he was more nervous, and that his arms, noticeably his right, were becoming stiff. He had choking spells while partaking of solid food, two or three times during a meal, and last July or August had one so bad that he nearly choked to death, and could not thereafter swallow for two weeks, when that power suddenly 
returned; even now his sister has to cut his food into fine particles so that he may chew and swallow it without danger. During the last four years he has remained in statu qno. There is no history of convulsions, and the development has been very slowly progressive.

At present he is not emaciated or anemic, weighs I 40

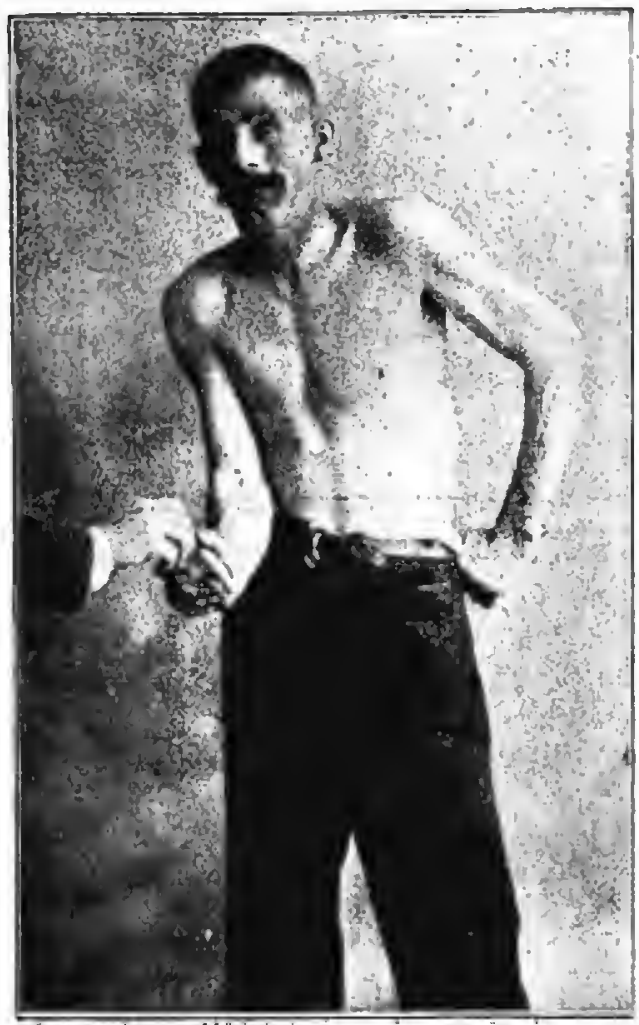

FIG. No. II.

pounds, and has a good color and appearance. Complains of cough, hoarseness, dyspnœa on exertion, difficulty in swallowing food, "getting stuck in his throat," and pain in the left arm at times; otherwise general health good as regards appetite, walking, working, and feelings generally. Sleeps well.

On examination (Photograph No. I.), it is noticed that 
he is restless and has no fever; pulse 80; no heart lesion; respirations, sitting, 22; lips bluish. Memory good, sight and hearing normal, no nystagmus, pupilliary, or eye muscle symptoms, optic nerves cloudy, particularly nasal side of left disk. When asked to put out his tongue, it appears as a short, thick, jerking, trembling mass, with general tremors, not fibrillary, running through it. $\mathrm{He}$ is unable to protrude it without jerking it in and out. Taste is normal. Speech is of a rapid, nasal, muffled character. He talks in a jerky whisper, as though he. were throwing the words out of his throat, and has difficulty in using his for phonating. On inspection, the pharynx and larynx are both seen to be intensely congested and sivollen; vocal corcls thickened and congested, but not paralyzed. The mucts membrane of the pharynx is perfectly insensible; touching it produces no gagging. The spasmodic condition of the muscles of the lips appears to give rise to the peculiar appearance or expression of the lower part of the face.

The right arm was first affected, and two years later the left. He is right-handed. Hands are unsteady and cannot be kept at rest on account of the marked rigid spastic condition, and general tremor "which is most marked in the right, and which is worse when "nervous" or noticed. When standing, arms are seen to hang partly flexed at elbow, and rotated inwards; as a rule, right hand hyper-extended and fingers flexed; left hand, wrist and fingers flexed; some irregular spasm of fingers with a remote resemblance to athetoid movements; no apparent loss of power as shown by work and unbuttoning clothing without difficulty, except for spasms present. Dynamometer, left, $55-45$; right $.55-55$. There is no paralysis.

In taking objects with his right hand, he first rotates the hand and takes the object from the under side, just the reverse of the way an object is usually taken. This appears to be his habitual way, but it is possible for him, with an effort, to take it in the natural way; when asked to do this, that is, take things from above (as we do), not below (see photo. No. II.), he.keeps the upper arm close to his side, whereas, when allowed to take them "his way," the elbow is held away from the body, as we naturally do. His grasp in both hands is shown to be very good, and there is no loss of sensibility. Apparently there is no atrophy nor gircle sensation. In the lower extremities nothing is 
noticeable except diminished reflexes. No indigestion; bowels irregular; no difficulty in holding or passing the urine.

Of course a number of conditions have been suggested, such as cerebro-glossoplegia or pseudo-bulbar paralysis, so-called Huntington's chorea, para-myo-clonus multiplex, lesions of both hemispheres, etc., but to none of which can this case, on close examination, be set down. The ultimate opinion of those who have given the case the closest study, is that it is probably due to some teratological defect of congenital origin.

Note on a Case of Astasia-Abasia.

D. V. Leblond (Journal de Médécine, December 25, I896) reports the case of a girl, nine years old, active, intelligent and without heredity nervous antecedents, who was suddenly attacked with severe pain in the legs, malaria fever and sore throat. The next day an eruption appeared on the legs resembling erythema nodosum, which was followed by slight cedema and diffuse redness. This disappeared with the pain, after rest in bed, and several doses of salicylate of sodium. As the patient was not suffering any more she asked to get up, but on attempting to do so she fell down. There was no vertigo or loss of consciousness, but an absolute impossibility to remain standing or take a step. When in bed all the movements of the lower extremities were preserved. There was no trouble of coordination of the upper extremities. A cold douche was followed by hysterical crisis, contraction of upper and lower extremities and loss of consciousness. A second one was better tolerated and later on when attempting to arise after the administration of a douche, the feet did not slip on touching the floor, but she was forced to hold on to the bed to remain standing. Little by little she risked making a step, holding firmly to the bed, and after half an hour's effort she succeeded in walking. This short history is interesting, owing to the mode of onset, in a child undoubtedly impressionable, but without nervous antecedents, and by the absence of hysterical stigmata. At first the diagnosis was difficult. The pain, subcutaneous nodes, œdema and angina, suggested rheumatism and a spinal complication. Without doubt there was absence of hysterical stigmata-no hysterical zone hemianæsthesia or narrowing of visual field-Astasia-abasia can, however, be a monosymptomatic manifestation of hysteria. Persuasion had no effect on this case, but isolation and separation from the parents during a short sojourn in the hospital sufficed with the douches to produce a positive cure which has persisted now for ten months.

FREEMAN. 